\title{
Legionella pneumonia in the Niagara Region, Ontario, Canada: a case series
}

Stephanie Cargnelli ${ }^{1}$, Jeff Powis ${ }^{2}$ and Jennifer L. Y. Tsang ${ }^{1,3^{*}}$

\begin{abstract}
Background: Legionella pneumophila, a major cause of Legionnaires' disease, accounts for 2-15\% of all communityacquired pneumonia requiring hospitalization and up to $30 \%$ of community-acquired pneumonia requiring intensive care unit admission. Early initiation of appropriate antimicrobial therapy is a crucial step in the prevention of morbidity and mortality. However, recognition of Legionnaires' disease continues to be challenging because of its nonspecific clinical features. We sought to describe hospitalized community-acquired Legionnaires' disease to increase awareness of this important and potentially lethal disease.

Methods: A retrospective multicenter observational study was conducted with all patients with confirmed Legionnaires' disease in the Niagara Region of the Province of Ontario, Canada, from June to December 2013.

Results: From June to December 2013, there were 14 hospitalized cases of Legionnaires' disease in the Niagara Region. Of these, $86 \%$ (12 patients) had at least one comorbidity and $71 \%$ (10 patients) were cigarette smokers. In our cohort, Legionnaires' disease was diagnosed with a combination of a urinary Legionella antigen test and a Legionella real-time polymerase chain reaction assay. Delay in effective antimicrobial therapy in the treatment of Legionella infection led to clinical deterioration. The majority of patients had met systemic inflammatory response syndrome criteria with fever $>38^{\circ} \mathrm{C}(71 \%)$, heart rate $>90$ beats per minute $(71 \%)$, and respiratory rate $>20$ breaths per minute (86\%). Eleven patients (79\%) required admission to the intensive care unit or step-down unit, and nine patients (64\%) required intubation. Clinical improvement after initiation of antimicrobials was protracted.

Conclusions: Legionnaires' disease should be considered during the late spring and summer months in patients with a history of tobacco use and various comorbidities. Clinically, patients presented with severe, nonspecific, multisystem disease characterized by shortness of breath, abnormal vital signs, and laboratory derangements including hyponatremia, elevated creatine kinase, and evidence of organ dysfunction. In addition, antimicrobial therapy with newer macrolides or respiratory fluoroquinolones should be initiated for severe community-acquired pneumonia requiring intensive care unit admission, prior to laboratory confirmation of diagnosis, especially when a clinical suspicion of Legionella infection exists.
\end{abstract}

Keywords: Legionella pneumophila, Legionnaires' disease, Community-acquired pneumonia

\section{Background}

Legionella pneumophila, a gram-negative bacteria and the main causative agent of legionellosis, was first recognized in 1976 [1]. Legionellosis has two distinct clinical presentations: (1) Pontiac fever, a self-limited, febrile, flulike illness;

\footnotetext{
* Correspondence: jennifer.tsang@mail.utoronto.ca

Jennifer L. Y. Tsang is the senior author.

${ }^{1}$ Michael G. DeGroote School of Medicine, Niagara Regional Campus, McMaster University, MDCL 3107, 1280 Main Street West, Hamilton, ON L8S 4K1, Canada

${ }^{3}$ Niagara Health, 1200 Fourth Avenue, St. Catharines, ON L2S OA9, Canada

Full list of author information is available at the end of the article
}

and (2) Legionnaires' disease. Legionnaires' disease is an atypical pneumonia that has clinical and radiographic findings similar to those of pneumococcal pneumonia [2-6]. It has an incubation period of 2-14 days. Patients might present with fever, cough, myalgia, asthenia, anorexia, and relative bradycardia [2]. Symptoms that are more suggestive of Legionnaires' disease include gastrointestinal symptoms (diarrhea, nausea, vomiting, and abdominal pain) and neurologic symptoms (headache, obtundation, seizures, and focal neurologic findings) [2, 7-10]. Legionnaires' disease is also characterized by nonspecific laboratory findings such 
as hyponatremia; hypophosphatemia; leukocytosis with relative lymphopenia; elevated creatine kinase, erythrocyte sedimentation rate, $\mathrm{C}$-reactive protein, and ferritin levels; myoglobulinuria; and microscopic hematuria [2].

Legionnaires' diseases accounts for $2-15 \%$ of hospitalized community-acquired pneumonia (CAP) and up to $30 \%$ of CAP requiring intensive care unit (ICU) admission $[11,12]$. The incidence of Legionnaires' disease rose by $192 \%$ in the United States between 2000 and 2009 to 11.5 cases per 1 million population [13]. Sixty-two percent of the cases occur in the summer and autumn seasons. This is due to the increased use of air-conditioning systems and cooling towers as well as to increased rainfall $[14,15]$. Approximately $20 \%$ of cases were travelassociated [16]. Only $4 \%$ of cases were associated with a known outbreak [2]. The risk factors for Legionnaires' disease include cardiopulmonary disease, cigarette smoking, age $>50$ years, diabetes, malignancy, and immunosuppressive state including glucocorticoid use [11, 17].

Legionnaires' disease causes significant morbidity and carries a mortality rate of 8-12\% [18]. Among patients who survive, recovery is often slow, and they are left with fatigue, neurologic and neuromuscular symptoms, and post-traumatic stress disorder [19]. Legionnaires' disease is also very costly, with a healthcare-associated cost of more than $\$ 23,000$ per case [20].

The diagnosis of Legionnaires' disease can be made using non-culture- and culture-based techniques [2]. A urinary antigen test that detects a component of the cell wall lipopolysaccharide is the first-line diagnostic test for Legionnaires' disease $[21,22]$. However, it detects only $L$. pneumophila serogroup 1 , which is the most virulent and most common cause of disease [11, 22, 23]. In Europe, $>90 \%$ of Legionnaires' disease cases were diagnosed by urinary antigen detection [2]. It is a rapid test (results available within hours), with a sensitivity of $56-99 \%$ [22], and it is positive within $48-72 \mathrm{~h}$ of symptom onset and can remain positive for several weeks or months despite treatment [21,22]. In addition to urinary antigen detection, molecular tests such as a realtime polymerase chain reaction (PCR) assay that targets the 23S-5S ribosomal RNA (rRNA) intergenic spacer region can be used for rapid detection, and this test has the capability of differentiating L. pneumophila from 50 non-L. pneumophila species [24].

The isolation of Legionella bacteria by culture of the lower respiratory tract using buffered charcoal yeast extract (BCYE) medium is still the gold standard for detecting Legionnaires' disease [22] because it allows the diagnosis of all Legionella species, outbreak investigation, further epidemiologic studies, and antimicrobial susceptibility testing [2]. However, culture of Legionella is very cumbersome and technically demanding, and the sensitivity is only around $20-80 \%[2,22]$.
Legionella species are intracellular pathogens; therefore, antibiotics should accumulate and be bioactive within the cells [2]. Azithromycin, doxycycline, or levofloxacin can be considered as first-line therapy [2]. It is recommended that levofloxacin $750 \mathrm{mg}$ daily for $5-10$ days or azithromycin $500 \mathrm{mg}$ daily for $3-5$ days be used in patients with Legionnaires' disease, except for patients with immunosuppression, severe disease, empyema, and extrapulmonary infection, as well as those undergoing inappropriate initial therapy, for whom an extended course is recommended [25-28]. Early initiation of appropriate therapy is important in reducing mortality associated with Legionnaires' disease [2, 29, 30].

In the summer of 2013, there were 14 hospitalized cases of Legionnaires' disease in the Niagara Region of the Province of Ontario, Canada. The primary aim of this study was to describe these14 cases of hospitalized community-acquired Legionnaires' disease to increase awareness of this important and potentially lethal disease.

\section{Methods \\ Study design}

We conducted a multicenter retrospective observational study of all patients with hospitalized Legionnaires' disease in the Niagara Region of the Province of Ontario, Canada, between June and December 2013. Legionnaires' disease was defined as a positive urinary Legionella antigen assay result in the presence of symptoms or Legionella real-time PCR of sputum or bronchoalveolar lavage (BAL) (both performed in the Public Health Ontario Laboratory). The urinary Legionella antigen assay was performed using a commercial Binax immunochromatographic test kits (Alere, Orlando, FL, USA) [31]. The results were reported as positive or negative. The Binax test has $>70 \%$ sensitivity and $>99 \%$ specificity for L. pneumophila serogroup 1 only [31]. The real-time PCR assay used in this study targets the 23S-5S rRNA intergenic spacer region. It has the ability to differentiate $L$. pneumophila from 50 other nonL. pneumophila species. The detailed protocol was described elsewhere [24]. All positive real-time PCR samples were cultured and identified to the genus, species, and serogroup levels using BCYE and buffered polymyxine $\mathrm{B}$, anisomycin, and vancomycin charcoal media. The plates were reincubated at $35{ }^{\circ} \mathrm{C}$ for up to 14 days and examined under a microscope daily for colonial morphology such as mottled surface and iridescent red-blue-green sheen or a faceted cut-glass appearance. Slide agglutination testing was used to speciate and serogroup L. pneumophila isolates and atypical Legionella-like isolates. Known antibodies were mixed with culture isolates on a glass microscope slide. Antibodies against 50 Legionella species/serogroup targets were used for this method on the basis of the protocol developed by the Centers for 
Disease Control and Prevention in the United States [32]. Agglutination reactions were scored on a scale from 4+ (strongest) to $1+$ (barely visible). In cases of cross-reaction (two or more species/serogroup targets reactive), results were compared against specific positive and negative controls. The strongest reaction was considered the final result.

\section{Data collection}

Our data collection form encompassed demographic data, epidemiologic data, medical history, symptoms, vital signs, and laboratory test results at admission. We also recorded daily laboratory test results, antimicrobial therapy information, and clinical outcome data when available. Data collection was conducted at three sites of Niagara Health where cases occurred, including the St. Catharines sites, the Greater Niagara General Hospital site, and the Welland site. Data collection was performed using electronic medical records (Meditech, Westwood, MA, USA; IMPAX, Agfa Health Care, Greenville, SC, USA) and paper medical charts. Approval with a waiver of informed consent for the study was obtained from the Niagara Health Research Ethics Board.

\section{Results}

\section{Demographics and diagnosis}

From June 2013 to December 2013, there were a total of 14 hospitalized Legionnaires' disease cases identified in the Niagara Region of Ontario, Canada. These contributed to approximately $2 \%$ of all hospitalized CAP. Of note, the average number of legionellosis cases reported by Niagara Health annually from 2011 to 2015 was 6 . Public Health Ontario performed a thorough evaluation of these cases and indicated that there were no epidemiological links.

Twelve (86 \%) of the fourteen Legionnaires' disease cases were identified with a positive urinary Legionella antigen assay. One case was tested negative with the urinary Legionella antigen assay due to non-L. pneumophila infection confirmed with BAL real-time PCR assay. One case did not have a urinary Legionella antigen assay sent but was tested positive for L. pneumophila with BAL realtime PCR assay. In total, seven (50\%) cases were tested positive with the real-time PCR assay, and three cases (21\%) were culture-positive (Table 1).

Demographics, medical comorbidities and risk factors are presented in Table 2 . The majority (12 patients, $86 \%$ ) of the patients had comorbidities, with hypertension, dyslipidemia, and diabetes mellitus occurring in more than half of the cohort. In addition to the presence of comorbidities, the other major risk factors for Legionnaires' disease in this patient population included cigarette smoking (ten patients [71\%]) and marijuana use (three patients [21\%]).

\section{Clinical features}

All 14 patients met systemic inflammatory response syndrome criteria (Table 2). Twelve patients (86 \%) reported cough, with $50 \%$ of the patients reporting sputum production. Only a minority of patients presented with gastrointestinal symptoms. Confusion or a change in mental status was present in $50 \%$ of patients at presentation.

Laboratory results were recorded for patients upon presentation to the emergency department and are provided in Table 2 . The most common laboratory abnormalities included an elevated leukocyte count (71\%), elevated creatine kinase (50\%), hyponatremia (64\%), elevated creatinine (64\%), elevated troponins (64\%), and elevated liver enzymes (86\%). At the time of admission, only five patients (36\%) had bilateral infiltrates visualized by chest $\mathrm{x}$-ray.

The Pneumonia Severity Index (PSI) is a prediction rule for prognosis that objectively stratifies patients with CAP into quintiles of risk for short-term mortality on the basis of 20 demographic and clinical variables routinely available at presentation. It has been validated with data on $>50,000$ patients [33]. PSI scores were calculated for each patient on admission (Table 3). The majority of patients had a PSI of grade IV or above (ten patients [71\%]).

\section{Treatment, clinical complications and outcomes}

The treatment, clinical complications, and outcomes of the 14 patients with Legionnaires' disease are summarized in Table 3. Delayed administration of appropriate antibiotic treatment with azithromycin or a fluoroquinolone (defined as not commencing antibiotics within $24 \mathrm{~h}$ after the patient presented to the emergency department) occurred in four patients. Eleven patients (79 \%) required admission to an ICU or a step-down unit. The step-down unit in Niagara Health is a monitored unit with a nurse-to-patient ratio of 1:2. All patients undergo continuous cardiac monitoring and oximetry. This unit manages patients who require noninvasive ventilatory support and vasopressive support. Nine patients (64\%) required mechanical ventilation, eight patients $(57 \%)$ required vasopressive support, and five patients (36 \%) required renal replacement therapy. Clinical improvement was protracted, with average ICU/step-down unit and hospital lengths of stay of 13 and 16 days, respectively. The hospital mortality rate was $21 \%$ in this cohort.

\section{Discussion}

Legionnaires' disease is a life-threatening condition that requires prompt diagnosis and treatment. In this study, $64 \%$ of the reported cases occurred from June to August 2013. This is typical in temperate climates in the northern hemisphere because the causative organism 
Table 1 Legionnaires' disease diagnostic tests

\begin{tabular}{|c|c|c|c|c|c|}
\hline Patient & Urinary antigen test & BAL real-time $P C R$ & BAL culture & Sputum real-time PCR & Sputum culture \\
\hline 1 & Positive & Negative & Negative & N/A & N/A \\
\hline 2 & N/A & Positive & Negative & N/A & N/A \\
\hline 3 & Positive & N/A & $\mathrm{N} / \mathrm{A}$ & N/A & N/A \\
\hline 4 & Positive & $\mathrm{N} / \mathrm{A}$ & $\mathrm{N} / \mathrm{A}$ & N/A & $N / A$ \\
\hline 5 & Positive & N/A & N/A & N/A & N/A \\
\hline 6 & Positive & Positive & Negative & N/A & N/A \\
\hline 7 & Positive & N/A & $\mathrm{N} / \mathrm{A}$ & N/A & N/A \\
\hline 8 & Positive & $N / A$ & N/A & Positive & Positive \\
\hline 9 & Positive & N/A & N/A & Positive & Positive \\
\hline 10 & Negative & Positive $^{a}$ & Negative & N/A & N/A \\
\hline 11 & Positive & $\mathrm{N} / \mathrm{A}$ & $\mathrm{N} / \mathrm{A}$ & Positive & Negative \\
\hline 12 & Positive & N/A & N/A & N/A & N/A \\
\hline 13 & Positive & $\mathrm{N} / \mathrm{A}$ & N/A & N/A & N/A \\
\hline 14 & Positive & Positive & Positive & N/A & N/A \\
\hline
\end{tabular}

N/A Not applicable, $B A L$ bronchoalveolar lavage, $P C R$ polymerase chain reaction

${ }^{a}$ Non-Legionella pneumophila

multiplies effectively in warm aquatic environments [2]. The median age of our patient cohort was 58 years, which was less than what was published by Public Health Ontario in 2011. Indeed, most of our patients were $<65$ years of age $(71 \%)$, despite the fact that the incidence of Legionnaires' disease is reported to increase with age [34]. This suggests that age may not be a prominent characteristic for community-acquired Legionnaires' disease that is not associated with a point source outbreak. Tobacco use is a well-documented risk factor for Legionnaires' disease [35], and our results showed that cigarette smoking was a common feature in our cohort of patients with Legionnaires' disease. A lessestablished risk factor for Legionnaires' disease is cannabis use. Results published by Nguyen et al. provided support for an increased risk of Legionnaires' disease in cannabis smokers and suggested that this risk may be cumulative with the risk incurred through tobacco use [36]. Interestingly, in our cohort of patients with Legionnaires' disease, we found that three patients were both cannabis and cigarette smokers.

Clinical recognition of Legionnaires' disease is made difficult by the fact that patient presentation is often inconsistent with the clinical presentation documented in the literature $[2,6]$. Our data suggest that the diagnosis of Legionnaires' disease on the basis of clinical presentation is unreliable.

Our results demonstrate that Legionnaires' disease results in severe pneumonia, as reflected by the high PSI score and impacts on multiple organ systems rather than being confined solely to the pulmonary system. The multisystem nature of Legionnaires' disease likely contributes in part to the acute presentation of patients with
Legionnaire's disease and the need for more intensive management. With almost three-fourths of the patients requiring admission to an ICU, almost two-thirds requiring mechanical ventilation for an average of 9 days, and over half requiring vasopressors, these results certainly corroborate the data suggesting that the clinical manifestations of Legionnaires' disease are often more severe than those of pneumonias caused by other infectious agents [37].

Chest $\mathrm{x}$-rays are one of the first diagnostic tests performed on patients upon arrival in the emergency department. As with much of the presentation thus far, the chest x-ray of patients with Legionnaires' disease does not possess any distinguishing features that would guide diagnosis. The initial chest $\mathrm{x}$-ray of just over one-third of the patients demonstrated bilateral airspace disease; the remaining two-thirds had unilateral findings. However, six of the patients with unilateral findings on their initial $\mathrm{x}$-ray progressed to bilateral findings on follow-up images. This progression of radiographic findings throughout hospital admission prior to noting improvements has been documented in the literature [6]. There were not any identifiable patterns in terms of proclivity for specific lobes or features of lung involvement.

The most common method of Legionnaires' disease diagnosis was urinary Legionella antigen testing (92.9 \%). In our case series, of the 13 patients tested for urinary Legionella antigen, 12 had a positive result. One of the patients was infected by non-L. pneumophila Legionella (diagnosed with BAL real-time PCR). As such, other forms of diagnostic tests, including real-time PCR and culture of sputum or BAL samples, are indicated if there is a suspicion of Legionnaires' disease and the result of 
Table 2 Characteristics, comorbidities, risk factors, symptoms, and vital signs

\begin{tabular}{|c|c|}
\hline Variable & Value \\
\hline Age, years, median (IQR) & $57(47-66)$ \\
\hline Male sex, $n(\%)$ & $8(57)$ \\
\hline \multicolumn{2}{|l|}{ Known comorbidities/risk factors, $n$ (\%) } \\
\hline Hypertension & $9(64)$ \\
\hline Dyslipidemia & $7(50)$ \\
\hline Diabetes & $4(29)$ \\
\hline Coronary artery disease & $3(21)$ \\
\hline Chronic lung disease & $2(14)$ \\
\hline Chronic renal insufficiency & $4(29)$ \\
\hline Immunosuppression & $2(14)$ \\
\hline Cigarette smoking & $10(71)$ \\
\hline Cannabis use & $3(21)$ \\
\hline \multicolumn{2}{|l|}{ Symptoms, n (\%) } \\
\hline Productive cough & $7(50)$ \\
\hline Nonproductive cough & $5(36)$ \\
\hline Shortness of breath & $12(86)$ \\
\hline Pleuritic chest pain & $3(21)$ \\
\hline Nonpleuritic chest pain & $2(14)$ \\
\hline Diarrhea & $4(29)$ \\
\hline Nausea/vomiting & $3(21)$ \\
\hline Abdominal pain & $4(29)$ \\
\hline Confusion/altered level of consciousness & $7(50)$ \\
\hline Headache & $3(21)$ \\
\hline Fatigue & $4(21)$ \\
\hline Rigors & $7(50)$ \\
\hline Myalgias & $4(29)$ \\
\hline \multicolumn{2}{|l|}{ Vital signs at triage, $n(\%)$} \\
\hline Heart rate $>90$ beats per minute & $10(71)$ \\
\hline Respiratory rate $>20$ breaths per minute & $12(93)$ \\
\hline Systolic blood pressure $<90 \mathrm{mmHg}$ & $1(7)$ \\
\hline Temperature $<36{ }^{\circ} \mathrm{C}$ or $>38^{\circ} \mathrm{C}$ & $10(71)$ \\
\hline \multicolumn{2}{|l|}{ Laboratory values (normal range), mean \pm SD } \\
\hline Leukocytes (4.0-11.0), $\times 10^{9} / \mathrm{L}$ & $14.9 \pm 4.9$ \\
\hline Creatinine (46-92), $\mu \mathrm{mol} / \mathrm{L}$ & $286 \pm 460$ \\
\hline Sodium (135-145), mmol/L & $133 \pm 4$ \\
\hline Phosphate $(0.8-1.45), \mathrm{mmol} / \mathrm{L}$ & $1.48 \pm 1.98$ \\
\hline Creatine kinase (40-200), U/L & $1914 \pm 2728$ \\
\hline Bilirubin (3-22), $\mu \mathrm{mol} / \mathrm{L}$ & $13 \pm 11$ \\
\hline Aspartate aminotransferase (10-40), U/L & $77 \pm 63$ \\
\hline Alanine aminotransferase (5-68), U/L & $54 \pm 27$ \\
\hline Alkaline phosphatase (50-135), U/L & $90 \pm 31$ \\
\hline Troponin $(<0.045), \mu \mathrm{g} / \mathrm{L}$ & $0.66 \pm 1.43$ \\
\hline Lactate $(0.4-2.1), \mathrm{mmol} / \mathrm{L}$ & $2.3 \pm 1.3$ \\
\hline
\end{tabular}

Table 2 Characteristics, comorbidities, risk factors, symptoms, and vital signs (Continued)

\begin{tabular}{ll}
\hline Arterial pH (7.35-7.45) & $7.32 \pm 0.14$ \\
Distribution of infiltrates on initial chest x-ray, $n(\%)$ & \\
Unilateral & $9(64)$ \\
Bilateral & $5(36)$ \\
\hline
\end{tabular}

urinary Legionella antigen testing is negative [38]. It has been suggested that a combination of urinary Legionella antigen testing and real-time PCR is the best initial approach to ensure detection of all Legionella infections and obtain results within a time frame capable of influencing management [39]. This is corroborated in our case series by the fact that one of the diagnoses would have been missed had a real-time PCR of a BAL sample not been done concurrently with urinary Legionella antigen testing.

Delay in initiating appropriate antibiotic therapy is known to be a poor prognostic factor in patients infected by Legionella [39]. In our case series, four patients had delayed initiation of antibiotic therapy with a fluoroquinolone or azithromycin. Although none of these patients died as a result of this delay in diagnosis and treatment, two of these patients had hospital stays that exceeded 25 days, and both of them required mechanical ventilation and vasopressor support. One patient also required dialysis throughout the admission, and three patients required admission to the ICU. As such, it is

Table 3 Treatment, clinical complications, and outcomes

\begin{tabular}{ll}
\hline Variable & Value \\
\hline Pneumonia Severity Index & $115 \pm 45$ \\
Score, mean \pm SD & $1(7)$ \\
Grade I, $n(\%)$ & $0(0)$ \\
Grade II, $n(\%)$ & $3(21)$ \\
Grade III, $n$ (\%) & $4(29)$ \\
Grade IV, $n$ (\%) & $6(43)$ \\
Grade V, $n(\%)$ & $4(29)$ \\
Delay in appropriate antimicrobial therapy, $n(\%)$ & \\
Clinical complications, $n$ (\%) & $8(57)$ \\
Vasopressor requirement & $9(64)$ \\
Mechanical ventilation & $5(36)$ \\
Renal replacement therapy & $11(79)$ \\
ICU/step-down unit admission & \\
Outcomes & $9 \pm 4$ \\
Duration of mechanical ventilation, days, mean \pm SD & $13 \pm 8$ \\
ICU/step-down unit length of stay, days, mean \pm SD & $16 \pm 10$ \\
Hospital length of stay, days, mean \pm SD & $3(21)$ \\
Hospital mortality, $n$ (\%) & \\
\hline ICU Intensive care unit &
\end{tabular}


evident that, while a delay in treatment did not result in a higher mortality rate, it has the potential to worsen disease severity and result in a need for more intensive management. Of note, the three patients who died were older (aged 66, 74, and 81 years, respectively). One patient was immunosuppressed on methotrexate and prednisone for rheumatoid arthritis; two patients had a significant cardiac history and diabetes; and two patients were cigarette smokers.

A study conducted in Switzerland demonstrated that patients infected with atypical pathogens, including Legionella, had clinical instability refractory to treatment with a $\beta$-lactam and required the addition of a macrolide to the treatment regimen [40]. The same study demonstrated that patients with more severe pneumonias benefited from a combination therapy as opposed to $\beta$-lactam monotherapy. As such, it is suggested that the initial treatment approach for patients presenting with severe CAP (those with a PSI category IV pneumonia) include a macrolide or fluoroquinolone with effectiveness in treating Legionella rather than waiting for laboratory confirmation of infection [40]. Our study suggests that patients presenting with severe multisystem disease during the summer or early autumn requiring admission to the ICU need atypical coverage with a macrolide or fluoroquinolone prior to laboratory confirmation. Atypical coverage should also be added to a treatment regimen when there is no improvement noted with $\beta$-lactam treatment.

Our study was limited by the fact that our data collection was dependent on the reporting and diagnostic tests ordered by other healthcare professionals. As a result, full patient histories and laboratory values could not be obtained for each patient. A prospective study would overcome this limitation. Last, we do not have a control group with non-Legionella CAP to assess risk factors for Legionnaires' disease.

\section{Conclusions}

Legionnaires' disease should be considered during the late spring and summer months in patients with a history of tobacco use and various comorbidities presenting with severe, multisystem disease characterized by shortness of breath; abnormal vital signs; and laboratory derangements, including hyponatremia, elevated creatine kinase, and evidence of organ dysfunction. Legionnaires' disease should be diagnosed with a combination of urinary Legionella antigen testing and Legionella real-time PCR if available. Antibiotic therapy with a macrolide or a fluoroquinolone should be initiated for severe CAP prior to laboratory confirmation of diagnosis, especially when a clinical suspicion exists.

\section{Abbreviations}

BAL: Bronchoalveolar lavage; BCYE: Buffered charcoal yeast extract; CAP: Community-acquired pneumonia; ICU: Intensive care unit;
PCR: Polymerase chain reaction; PSI: Pneumonia Severity Index; rRNA: Ribosomal RNA

\section{Acknowledgements}

JLYT holds a McMaster University Department of Medicine Internal Career Research Award. We acknowledge Allison Brown and Dr. Matthew Greenway for their assistance in obtaining research ethics board approval. We also acknowledge Virginia Pullar, Kathleen Willis, and Phillip Guy for their efforts in obtaining the medical records of patients included in this study. Finally, we would like to thank the medical records staff of the Niagara Health System for locating the charts for review.

\section{Funding}

No funding was obtained for this study.

Availability of data and materials

All relevant raw data are available for sharing upon request.

\section{Authors' contributions}

SC made contributions to study conception and design, acquisition of data, analysis and interpretation of data, and the drafting of the manuscript. JP was involved in the critical review of the manuscript for important intellectual content. JLYT made contributions to study conception and design, acquisition of data, analysis and interpretation of data, and the drafting and revision of the manuscript. All authors read and approved the final manuscript.

\section{Competing interests}

The authors declare that they have no competing interests.

Consent for publication

Not applicable.

Ethics approval and consent to participate

Ethics approval for the study was obtained from the Niagara Health System Research Ethics Board. The need for consent was waived. The reference number for the study is 2014-03-001.

\section{Author details}

${ }^{1}$ Michael G. DeGroote School of Medicine, Niagara Regional Campus, McMaster University, MDCL 3107, 1280 Main Street West, Hamilton, ON L8S 4K1, Canada. ${ }^{2}$ Toronto East General Hospital, 825 Cowell Avenue, Toronto, ON M4C 3E7, Canada. ${ }^{3}$ Niagara Health, 1200 Fourth Avenue, St. Catharines, ON L2S OA9, Canada.

Received: 7 May 2016 Accepted: 19 October 2016

Published online: 01 December 2016

References

1. Fraser DW, Tsai TR, Orenstein W, Parkin WE, Beecham HJ, Sharrar RG, et al. Legionnaires' disease. N Engl J Med. 1977;297:1189-97.

2. Cunha BA, Burillo A, Bouza E. Legionnaires' disease. Lancet. 2016;387:376-85.

3. Cunha BA. Clinical features of Legionnaires' disease. Semin Respir Infect. 1998;13:116-27.

4. Cunha BA. Severe Legionella pneumonia: rapid presumptive clinical diagnosis with Winthrop-University Hospital's weighted point score system (modified). Heart Lung. 2008;37:311-20. A published erratum appears in Heart Lung. 2008;37:403.

5. Cunha BA. Legionnaires' disease: clinical differentiation from typical and other atypical pneumonias. Infect Dis Clin North Am. 2010;24:73-105.

6. Mulazimoglu L, Yu VL. Can Legionnaires disease be diagnosed by clinical criteria? A critical review. Chest. 2001;120:1049-53.

7. Kirby BD, Snyder KM, Meyer RD, Finegold SM. Legionnaires' disease: report of sixty-five nosocomially acquired cases and review of the literature. Medicine (Baltimore). 1980;59:188-205.

8. Granados A, Podzamczer D, Gudiol F, Manresa F. Pneumonia due to Legionella pneumophila and pneumococcal pneumonia: similarities and differences on presentation. Eur Respir J. 1989:2:130-4.

9. Roig J, Aguilar X, Ruiz J, Domingo C, Mesalles E, Manterola J, et al. Comparative study of Legionella pneumophila and other nosocomial-acquired pneumonias. Chest. 1991;99:344-50. 
10. Yu VL, Kroboth FJ, Shonnard J, Brown A, McDearman S, Magnussen M Legionnaires' disease: new clinical perspective from a prospective pneumonia study. Am J Med. 1982;73:357-61.

11. Stout JE, Yu VL. Legionellosis. N Engl J Med. 1997;337:682-7.

12. Woodhead MA, Macfarlane JT, McCracken JS, Rose DH, Finch RG. Prospective study of the aetiology and outcome of pneumonia in the community. Lancet. 1987;1:671-4

13. Centers for Disease Control and Prevention (CDC). Legionellosis-United States, 2000-2009. MMWR Morb Mortal Wkly Rep. 2011;60:1083-6.

14. Fisman DN, Lim S, Wellenius GA, Johnson C, Britz P, Gaskins M, et al. It's not the heat, it's the humidity: wet weather increases legionellosis risk in the greater Philadelphia metropolitan area. J Infect Dis. 2005;192:2066-73.

15. Hicks LA, Rose Jr CE, Fields BS, Drees ML, Engel JP, Jenkins PR, et al. Increased rainfall is associated with increased risk for legionellosis. Epidemiol Infect. 2007:135:811-7.

16. Beauté J, Zucs P, de Jong B. Risk for travel-associated Legionnaires' disease, Europe, 2009. Emerg Infect Dis. 2012;18:1811-6.

17. Winn Jr WC. Legionnaires disease: historical perspective. Clin Microbiol Rev. 1988;1:60-81.

18. Dominguez A, Alvarez J, Sabria M, Carmona G, Torner N, Oviedo M, et al. Factors influencing the case-fatality rate of Legionnaires' disease. Int J Tuberc Lung Dis. 2009;13:407-12.

19. Lettinga KD, Verbon A, Nieuwkerk PT, Jonkers RE, Gersons BPR, Prins JM, et al. Health-related quality of life and posttraumatic stress disorder among survivors of an outbreak of Legionnaires disease. Clin Infect Dis. 2002;35:11-7.

20. Vinson NG. Towards estimating the economic burden of waterborne illness in Canada: what do we know, where do we go? Poster presented at The Ontario Public Health Convention (TOPHC), Toronto, ON, Canada, 2-4 Apr 2012.

21. Shimada T, Noguchi Y, Jackson JL, Miyashita J, Hayashino Y, Kamiya T, et al. Systematic review and metaanalysis: urinary antigen tests for legionellosis. Chest. 2009;136:1576-85.

22. Jarraud S, Descours G, Ginevra C, Lina G, Etienne J. Identification of Legionella in clinical samples. Methods Mol Biol. 2013;954:27-56.

23. Helbig JH, Uldum SA, Bernander S, Lück PC, Wewalka G, Abraham B, et al. Clinical utility of urinary antigen detection for diagnosis of communityacquired, travel-associated, and nosocomial Legionnaires' disease. J Clin Microbiol. 2003;41:838-40.

24. Yang G, Benson R, Pelish T, Brown E, Winchell JM, Fields B. Dua detection of Legionella pneumophila and Legionella species by real-time PCR targeting the 23S-5S rRNA gene spacer region. Clin Microbiol Infect. 2010;16:255-61.

25. Mandell LA, Wunderink RG, Anzueto A, Bartlett JG, Campbell GD, Dean NC, et al. Infectious Diseases Society of America/American Thoracic Society consensus guidelines on the management of community-acquired pneumonia in adults. Clin Infect Dis. 2007;44 Suppl 2:S27-72.

26. Pedro-Botet ML, Yu VL. Treatment strategies for Legionella infection. Expert Opin Pharmacother. 2009;10:1109-21.

27. Yu VL, Greenberg RN, Zadeikis N, Stout JE, Khashab MM, Olson WH, et al. Levofloxacin efficacy in the treatment of community-acquired legionellosis. Chest. 2004;125:2135-9.

28. Kuzman I, Soldo I, Schönwald S, Culig J. Azithromycin for treatment of community acquired pneumonia caused by Legionella pneumophila: a retrospective study. Scand J Infect Dis. 1995;27:503-5.

29. Heath $\mathrm{CH}$, Grove DI, Looke DF. Delay in appropriate therapy of Legionella pneumonia associated with increased mortality. Eur J Clin Microbiol Infect Dis. 1996;15:286-90.

30. Gudiol C, Verdaguer R, Angeles Domínguez M, Fernández-Sevilla A, Carratalà J. Outbreak of Legionnaires' disease in immunosuppressed patients at a cancer centre: usefulness of universal urine antigen testing and early levofloxacin therapy. Clin Microbiol Infect. 2007;13:1125-8.

31. Kazandjian D, Chiew R, Gilbert GL. Rapid diagnosis of Legionella pneumophila serogroup 1 infection with the Binax enzyme immunoassay urinary antigen test. J Clin Microbiol. 1997;35:954-6.

32. Wilkinson HW, Fikes BJ. Slide agglutination test for serogrouping Legionella pneumophila and atypical Legionella-like organisms. J Clin Microbiol. 1980;11:99-101.

33. Aujesky D, Fine MJ. The Pneumonia Severity Index: a decade after the initial derivation and validation. Clin Infect Dis. 2008;47 Suppl 3:S133-9.

34. Silk BJ, Foltz JL, Ngamsnga K, Brown E, Muñoz MG, Hampton LM, et al. Legionnaires' disease case-finding algorithm, attack rates, and risk factors during a residential outbreak among older adults: an environmental and cohort study. BMC Infect Dis. 2013;13:291.
35. Bagaitkar J, Demuth DR, Scott DA. Tobacco use increases susceptibility to bacterial infection. Tob Induc Dis. 2008:4:12

36. Nguyen LT, Picard-Bernard V, Perriot J. Legionnaires disease in cannabis smokers. Chest. 2010;138:989-91.

37. Vergis EN, Akbas E, Yu VL. Legionella as a cause of severe pneumonia. Semin Respir Crit Care Med. 2000;21:295-304.

38. Reller LB, Weinstein MP, Murdoch DR. Diagnosis of Legionella infection. Clin Infect Dis. 2003;36:64-9.

39. Roig J, Rello J. Legionnaires' disease: a rational approach to therapy. J Antimicrob Chemother. 2003;51:1119-29.

40. Garin N, Genné D, Carballo S, Chuard C, Eich G, Hugli O, et al. $\beta$-Lactam monotherapy vs $\beta$-lactam-macrolide combination treatment in moderately severe community-acquired pneumonia: a randomized noninferiority trial. JAMA Intern Med. 2014;174:1894-901.

\section{Submit your next manuscript to BioMed Central and we will help you at every step:}

- We accept pre-submission inquiries

- Our selector tool helps you to find the most relevant journal

- We provide round the clock customer support

- Convenient online submission

- Thorough peer review

- Inclusion in PubMed and all major indexing services

- Maximum visibility for your research

Submit your manuscript at www.biomedcentral.com/submit
) Biomed Central 